\title{
Multiple oncogenic mutations related to targeted therapy in nasopharyngeal carcinoma
}

\author{
Jian-Wei Zhang ${ }^{1,2+}$, Tao Qin ${ }^{1,2+}$, Shao-Dong Hong ${ }^{1,2+}$, Jing Zhang ${ }^{1,2}$, Wen-Feng Fang ${ }^{1,2}$, Yuan-Yuan Zhao ${ }^{1,2}$, \\ Yun-Peng Yang ${ }^{1,2}$, Cong Xue ${ }^{1,2}$, Yan Huang ${ }^{1,2}$, Hong-Yuan Zhao ${ }^{1,2}$, Yu-Xiang Ma ${ }^{1,2}$, Zhi-Huang Hu ${ }^{1,2}$, \\ Pei-Yu Huang ${ }^{1,3}$ and Li Zhang ${ }^{1,2^{*}}$
}

\begin{abstract}
Introduction: An increasing number of targeted drugs have been tested for the treatment of nasopharyngeal carcinoma (NPC). However, targeted therapy-related oncogenic mutations have not been fully evaluated. This study aimed to detect targeted therapy-related oncogenic mutations in NPC and to determine which targeted therapy might be potentially effective in treating NPC.

Methods: By using the SNaPshot assay, a rapid detection method, 19 mutation hotspots in 6 targeted therapy-related oncogenes were examined in 70 NPC patients. The associations between oncogenic mutations and clinicopathologic factors were analyzed.

Results: Among 70 patients, 12 (17.1\%) had mutations in 5 oncogenes: 7 (10.0\%) had v-kit Hardy-Zuckerman 4 feline sarcoma viral oncogene homolog (KIT) mutation, 2 (2.8\%) had epidermal growth factor receptor (EGFR) mutation, 1 (1.4\%) had phosphatidylinositol-4,5-bisphosphate 3-kinase, catalytic subunit alpha (PIK3CA) mutation, 1 (1.4\%) had Kirsten rat sarcoma viral oncogene homolog (KRAS) mutation, and 1 (1.4\%) had simultaneous EGFR and v-Raf murine sarcoma viral oncogene homolog B1 (BRAF) mutations. No significant differences were observed between oncogenic mutations and clinicopathologic characteristics. Additionally, these oncogenic mutations were not associated with tumor recurrence and metastasis.
\end{abstract}

Conclusions: Oncogenic mutations are present in NPC patients. The efficacy of targeted drugs on patients with the related oncogenic mutations requires further validation.

Keywords: Nasopharyngeal carcinoma, Oncogenic mutation, SNaPshot

\section{Background}

Nasopharyngeal carcinoma (NPC) is characterized by a unique geographic distribution [1]. The incidence of NPC in South China is one of the highest worldwide (20 to 30 per 100,000) [2]. Radiotherapy with or without chemotherapy is the standard of care for non-metastatic disease [3]. With the development of radiotherapy techniques, the prognosis of early-stage NPC has been obviously improved, as indicated by a 5 -year local

\footnotetext{
* Correspondence: zhangli6@mail.sysu.edu.cn

${ }^{\dagger}$ Equal contributors

'Sun Yat-sen University Cancer Center; State Key Laboratory of Oncology in South China; Collaborative Innovation Center for Cancer Medicine,

Guangzhou 510060, Guangdong, P. R. China

2Department of Medical Oncology, Sun Yat-sen University Cancer Center, No. 651 Dongfeng Road East, Guangzhou 510060, Guangdong, P. R. China Full list of author information is available at the end of the article
}

control rate of $95 \%$ and a 5-year disease-free survival rate of $77 \%$ [4]. Distant metastasis, which occurred in approximately $20 \%$ of patients with locally advanced disease, was the main reason for treatment failure [4]. For patients with metastatic disease, the median overall survival (OS) was less than 2 years.

Currently, with the rapid development of molecular medicine, an increasing number of targeted drugs have been developed and used in cancer treatment, thereby providing new options for the management of NPC. Several clinical trials testing targeted therapy in NPC demonstrated the promising efficacy of targeted drugs such as cetuximab [5], bevacizumab [6], and sorafenib [7]. However, none of these targeted drugs have been approved by the Food and Drug Administration (FDA) for use in NPC due to the limited improvement on survival.

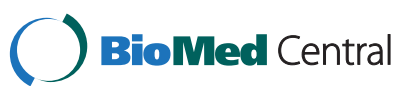

(c) 2015 Zhang et al.; licensee BioMed Central. This is an Open Access article distributed under the terms of the Creative Commons Attribution License (http://creativecommons.org/licenses/by/4.0), which permits unrestricted use, distribution, and reproduction in any medium, provided the original work is properly credited. The Creative Commons Public Domain Dedication waiver (http://creativecommons.org/publicdomain/zero/1.0/) applies to the data made available in this article unless otherwise stated. 
Table 1 Clinical characteristics of 70 patients with nasopharyngeal carcinoma (NPC)

\begin{tabular}{lc}
\hline Characteristic & Number of patients (\%) \\
\hline Sex & $46(65.7)$ \\
Man & $24(34.3)$ \\
Women & \\
Smoking status & $29(41.4)$ \\
Smoker & $41(49.2)$ \\
Non-smoker & \\
Clinical stage & $2(2.9)$ \\
I & $14(20.0)$ \\
॥ & $39(55.7)$ \\
III & $15(21.4)$ \\
IV & \\
EBV copy number & $59(84.3)$ \\
$>1,000$ & $11(15.7)$ \\
$<1,000$ & \\
Therapy & $1(1.4)$ \\
No treatment & $16(22.9)$ \\
Radiotherapy & $53(75.7)$ \\
Chemoradiotherapy & \\
Recurrence or metastasis & $11(15.7)$ \\
Yes & $59(84.3)$ \\
No & \\
\hline EBV Epstein-Barr virus. & \\
\hline
\end{tabular}

EBV, Epstein-Barr virus.

In fact, not all of the patients could benefit from targeted therapy. The sensitivity of targeted drugs is related to the genetic makeup of individual tumors; therefore, the identification of somatic mutations is increasingly important in the clinical management of cancer [8].

Different types of targeted drugs have been tested in clinical trials for NPC, including monoclonal antibodies against epidermal growth factor receptor (EGFR) and vascular endothelial growth factor (VEGF) as well as small-molecule tyrosine kinase inhibitors (TKIs) [5-7]. These drugs had different mechanisms of inhibiting molecular signaling pathways. Monoclonal antibodies against EGFR, such as cetuximab and nimotuzumab, bind to EGFR to block its downstream signaling pathway. Additionally, the monoclonal antibody against VEGF had anti-angiogenic effects. Among small-molecule TKIs, such as sorafenib, famitinib, and dasatinib, most were multi-targeted TKIs. However, until now, few studies have focused on the roles of oncogenic mutations in NPC, and the results are controversial. No specific oncogenic target has yet been identified in NPC for targeted therapy [9].

Here, we aimed to evaluate the prevalence of targeted therapy-related oncogenic mutations in NPC using the
SNaPshot assay and to identify potentially effective targets in NPC. We mainly focused on the EGFR signaling pathway components, including EGFR, phosphatidylinositol-4,5bisphosphate 3-kinase, catalytic subunit alpha (PIK3CA), Kirsten rat sarcoma viral oncogene homolog (KRAS), and v-Raf murine sarcoma viral oncogene homolog B1 $(B R A F)$. In addition, the v-kit Hardy-Zuckerman 4 feline sarcoma viral oncogene homolog (KIT) gene was also analyzed. All of these factors are common hotspots for mutations in cancer.

\section{Methods \\ Patient selection}

Consecutive patients who were pathologically diagnosed with NPC between April 2012 and December 2012 at the Sun Yat-sen University Cancer Center (Guangzhou, China) for whom fresh-frozen tissue samples were available were enrolled. The clinicopathologic information of all patients was collected, including sex, age, tumor stage, pathologic type, and treatment regimen. Tumor stage was classified according to the Union for International Cancer Control (UICC) 2010 NPC TNM staging system. Fresh nasopharyngeal tissue samples were obtained from all patients. Informed consent forms outlining tissue collection and clinical information analyses were signed by all patients. The protocol was approved by the institutional ethics committee of the Sun Yat-sen University Cancer Center.

\section{DNA extraction}

Fresh frozen tissues confirmed to be NPC by hematoxylin and eosin staining were obtained and stored in microtubes at $-80^{\circ} \mathrm{C}$. DNA was extracted by using the QIAamp DNA extraction kit (QIAGEN, Shanghai, China) according to the manufacturer's protocol. Briefly, these tissues were transferred to 1.5-mL Eppendorf tubes, treated with animal tissue lysis buffer, and incubated with proteinase $\mathrm{K}$ at $56^{\circ} \mathrm{C}$ overnight. After tissue lysis, $200 \mu \mathrm{L}$ alkaline lysis buffer and $200 \mu \mathrm{L}$ ethanol were added. The lysate was then transferred to the QIAamp MinElute column, $20 \mu \mathrm{L}$ elution buffer was added, and the DNA was collected.

\section{SNaPshot assay}

Multiple oncogenic mutations were detected with the SNaPshot multiplex kit (QIAGEN, Shanghai, China), which involves multiplex polymerase chain reaction (PCR), multiplexed single-base primer extension, and capillary electrophoresis. The experiment was performed according to the instructions of the kit. Five oncogenes, EGFR, PIK3CA, $K R A S, B R A F$, and $K I T$, were detected, which were mainly involved in the EGFR signaling pathway. The SNaPshot assay is more flexible and slightly more sensitive than direct sequencing $[10,11]$. 


\section{Statistical analysis}

The associations between oncogenic mutations and clinicopathologic parameters were assessed with Fisher's exact test or Student's $t$ test. A $P$ value of less than 0.05 was considered significant. SPSS software 16.0 (SPSS Inc., Chicago, IL, USA) was used in the statistical analysis.

\section{Results}

\section{Patient characteristics}

A total of 70 patients were enrolled for the analysis. Among the 70 patients, 46 were men and 24 were women, with a median age of 46 years (range, 24 to 73 years). According to the WHO histological classification, all patients were diagnosed with non-keratinizing undifferentiated carcinoma. The median follow-up duration was 25.8 months, with January 2014 as the last follow-up date. Only 11 patients had tumor recurrence or metastasis. The baseline characteristics of all patients are shown in Table 1.

\section{Oncogenic mutation profiles}

Nineteen mutation hotspots of 5 targeted therapy-related oncogenes were examined in 70 NPC patients. Among the 70 patients, $12(17.1 \%)$ had mutations in 5 oncogenes: 7 (10.0\%) had KIT (M541L) mutation, 2 (2.8\%) had EGFR (T790M) mutation, 1 (1.4\%) had PIK3CA (H1047R) mutation, 1 (1.4\%) had KRAS (G13D) mutation, and 1 (1.4\%) had simultaneous EGFR and BRAF (V600E) mutations (Figures 1 and 2).

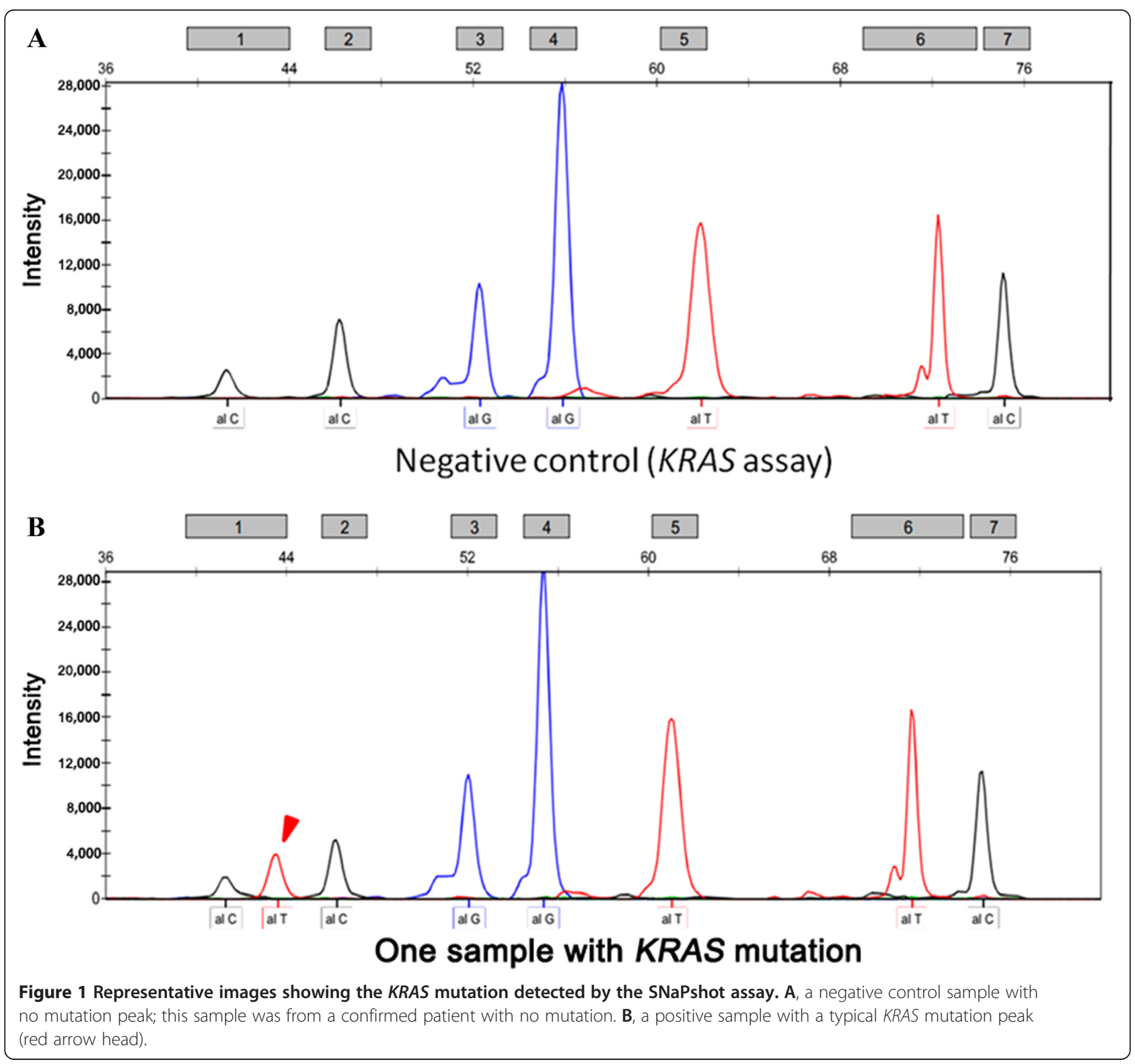




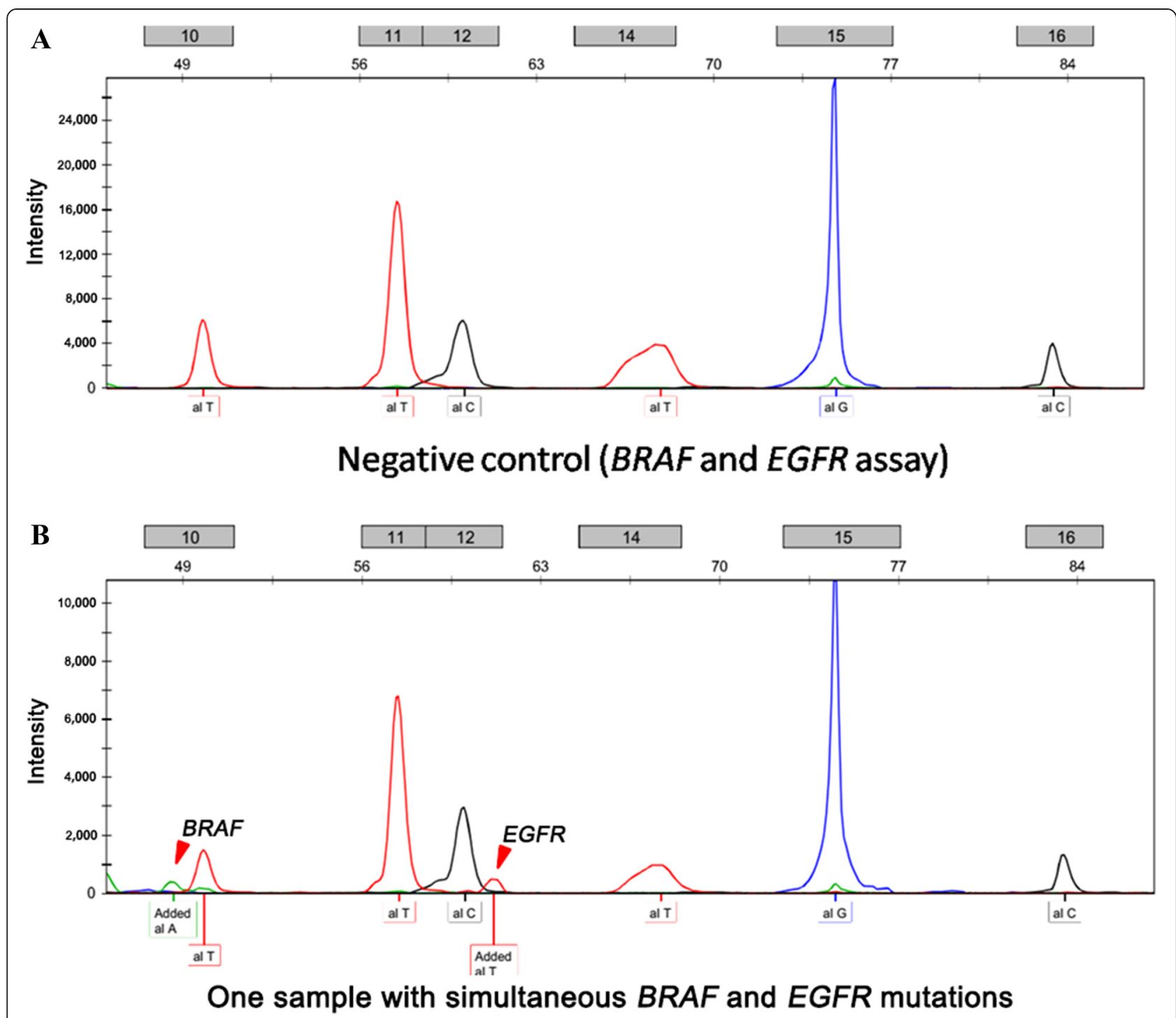

Figure 2 Representative images showing simultaneous BRAF and EGFR mutations detected by the SNaPshot assay. A, a negative control sample with no mutation peak; this sample was from a confirmed patient with no mutations. B, a positive sample with typical BRAF and EGFR mutation peaks (red arrow heads).

\section{Associations between oncogenic mutations and clinicopathologic characteristics}

Between the mutation and wild-type groups, no significant differences were observed for the clinicopathologic factors, including age, sex, clinical stage, EBV copy number, and recurrence/metastasis (Table 2). Only 11 patients had disease recurrence and metastasis; hence, survival analysis was not performed in this study.

\section{Discussion}

In this study, with the rapid detection method, we found that KIT mutation was the most frequent in NPC followed by EGFR mutation, whereas PI3KCA, KRAS, and $B R A F$ mutations were less frequent, which was consistent with the results of a previous study [12].
No obvious association was found between oncogenic mutations and clinicopathologic factors. Although another retrospective study showed that NPC patients who had recurrence or developed metastases had high mutation frequencies due to the relatively short follow-up duration, our study did not find the same result.

KIT is a transmembrane tyrosine kinase receptor encoded by the proto-oncogene C-KIT. The activation of KIT is associated with tumor proliferation, differentiation, adhesion, and apoptosis [13]. KIT was reported to be expressed in 33\%-88\% of NPCs [14-16]. Additionally, KIT mutation was also reported in our previous study [17]. It is well known that the KIT oncogene inhibitor imatinib has been widely used in clinical practice and has been approved for use in melanoma, gastrointestinal stromal 
Table 2 Associations between oncogenic mutations and clinical characteristics of NPC

\begin{tabular}{|c|c|c|c|}
\hline Parameter & Mutation group & Wild-type group & $P$ \\
\hline Total & 12 & 58 & \\
\hline Age (years) & & & 0.342 \\
\hline$>46$ & 4 & 31 & \\
\hline$\leq 46$ & 8 & 27 & \\
\hline Sex & & & 0.526 \\
\hline Man & 9 & 37 & \\
\hline Women & 3 & 21 & \\
\hline Clinical stage & & & 1.00 \\
\hline $1+\|$ & 3 & 13 & \\
\hline$I I I+I V$ & 9 & 45 & \\
\hline EBV copy number & & & 0.092 \\
\hline$>1,000$ & 11 & 38 & \\
\hline$<1,000$ & 1 & 20 & \\
\hline Recurrence or metastasis & & & 0.675 \\
\hline Yes & 1 & 10 & \\
\hline No & 11 & 48 & \\
\hline
\end{tabular}

The Fisher's exact test was performed in this analysis.

tumor (GIST), and chronic myelogenous leukemia (CML) patients with KIT mutations [18,19]. In our study, a KIT mutation in exon 10 (M541L), which is different than the functional mutations in exon 9 and exon 11 [20], occurred in $10 \%$ of NPC patients. However, the KIT (M541L) mutation was considered a gene polymorphism in CML. A myeloid cell line with the KIT (M541L) mutation was sensitive to imatinib in preclinical studies [21,22]. In addition, in previous experiments, imatinib could inhibit the proliferation of NPC cell lines (CEN-1, CNE2, Hone-1 C-666, SUNE-1, and 5-8 F) in a dose-dependent manner [17]. Thus, whether imatinib could be used in NPC patients with KIT mutations remains unknown, and further clinical trials are necessary to validate the efficacy of imatinib for treating NPC patients with KIT mutations.

EGFR is also a transmembrane tyrosine kinase receptor. EGFR overexpression in NPC is quite common and has been reported with a prevalence as high as $80 \%$ in primary NPC biopsies [23-25]. It has been proven that EGFR overexpression was indicative of poor prognosis and was associated with a low local control rate and short survival [26,27]. Thus, EGFR is considered an important target in NPC. However, EGFR mutations are reported to have an extremely low prevalence $(0-1 \%)$ in NPC [28,29]. In our study, EGFR mutations were detected in $3(4.3 \%)$ patients, all of whom had the T790M mutation in exon 20. In a phase II clinical trial of gefitinib for treating NPC, there was no response. The median progression-free survival (PFS) and OS were 4 and 16 months, respectively [30]. This result could be explained by the low EGFR mutation rate in NPC. In addition, patients with the T790M mutation showed primary resistance to gefitinib [31]. However, a new generation of EGFR TKIs, including AZD9291 and CO-1686, that target T790M has been recently developed $[32,33]$. Perhaps a small subset of NPC patients could benefit from these new EGFR TKIs.

Phosphatidylinositol 3 kinase (PI3K) is involved in diverse cellular functions, including cell proliferation, differentiation, motility, and survival. The PI3K-AKT pathway plays an important role in different tumor types. Activation of the PI3K pathway is significant in the development of NPC [34,35]. The PI3KCA mutation rate was low. In the present study, only 1 patient (1.4\%) had PI3KCA (H1047Y) mutation. The treatment of NPC cells with the PI3K inhibitor LY294002 led to the inhibition of AKT activation [36]. In addition, another inhibitor, MK-2206, which targets AKT, abrogates the AKT signaling [37]. These drugs are currently being evaluated in clinical trials. The PI3K-AKT pathway might be a new target in NPC [29].

The RAS/RAF/ERK pathway, which also plays an important role in tumor development, is downstream of EGFR [38,39]. Previous studies detected no KRAS mutations in NPC specimens or cell lines $[29,40]$. In the present study, KRAS mutations were detected in only 1 patient. Mutations of the Ras signaling pathway might lead to cetuximab resistance in NPC [41]. Furthermore, the BRAF (V600E) mutation was detected in only 1 patient. BRAF mutations were more common in melanoma, and a BRAF inhibitor has been approved for the treatment of melanoma. Although the mutation rate is not high, $B R A F$ is also a candidate biomarker for targeted therapy.

This study has several limitations. First, only 5 oncogenes related to the available targeted drugs were selected in our study. Second, the follow-up duration was short, and survival analysis was not conducted. Third, compared with deep sequencing, the SNaPshot method provides high-throughput data and is less expensive; however, it was not adequate to detect all genes in NPC. Therefore, our team is preparing to conduct whole-genome sequencing for NPC, which might provide more molecular information for NPC in the near future.

\section{Conclusions}

In our study, we found that $10 \%$ of patients with NPC had KIT mutations that were not at the commonly mutated sites. Additionally, other hotspot oncogenic mutations were infrequent in NPC patients from South China. The discovery of new therapeutic targets is underway.

\section{Competing interests}

The authors declare that they have no competing interests. 


\section{Authors' contributions}

Conceived and designed the experiments: LZ. Performed the experiments: JWZ, TQ, and SDH. Analyzed the data: JWZ, TQ, and SDH. Contributed clinical sample/materials/analysis tools: JZ, WFF, YYZ, YPY, CX, YH, HYZ, YXM, $\mathrm{ZHH}, \mathrm{PYH}$. Wrote the manuscript: JWZ, TQ, and SDH. All authors read and approved the final manuscript.

\section{Acknowledgements}

This study was supported in part by a grant from the National High Technology Research and Development Program of China (No. 2012AA02A501)

\section{Author details}

'Sun Yat-sen University Cancer Center; State Key Laboratory of Oncology in South China; Collaborative Innovation Center for Cancer Medicine, Guangzhou 510060, Guangdong, P. R. China. ²Department of Medical Oncology, Sun Yat-sen University Cancer Center, No. 651 Dongfeng Road East, Guangzhou 510060, Guangdong, P. R. China. ${ }^{3}$ Department of Nasopharyngeal Carcinoma, Sun Yat-sen University Cancer Center, Guangzhou 510060, Guangdong, P. R. China.

\section{Received: 10 October 2014 Accepted: 9 February 2015} Published online: 08 April 2015

\section{References}

1. Chan AT, Gregoire V, Lefebvre JL, Licitra L, Hui EP, Leung SF, et al. Nasopharyngeal cancer: EHNS-ESMO-ESTRO Clinical Practice Guidelines for diagnosis, treatment and follow-up. Ann Oncol. 2012;23:183-5.

2. Cao SM, Simons MJ, Qian CN. The prevalence and prevention of nasopharyngeal carcinoma in China. Chin J Cancer. 2011;30:114-9.

3. Ma BB, Hui EP, Chan AT. Systemic approach to improving treatment outcome in nasopharyngeal carcinoma: current and future directions. Cancer Sci. 2008:99:1311-8.

4. Xiao WW, Huang SM, Han F, Wu SX, Lu LX, Lin CG, et al. Local control, survival, and late toxicities of locally advanced nasopharyngeal carcinoma treated by simultaneous modulated accelerated radiotherapy combined with cisplatin concurrent chemotherapy: long-term results of a phase 2 study. Cancer. 2011;117:1874-83.

5. Ma BB, Kam MK, Leung SF, Hui EP, King AD, Chan SL, et al. A phase II study of concurrent cetuximab-cisplatin and intensity-modulated radiotherapy in locoregionally advanced nasopharyngeal carcinoma. Ann Oncol. 2012;23:1287-92.

6. Lee NY, Zhang Q, Pfister DG, Kim J, Garden AS, Mechalakos J, et al. Addition of bevacizumab to standard chemoradiation for locoregionally advanced nasopharyngeal carcinoma (RTOG 0615): a phase 2 multi-institutional trial. Lancet Oncol. 2012;13:172-80.

7. Xue C, Huang Y, Huang PY, Yu QT, Pan JJ, Liu LZ, et al. Phase II study of sorafenib in combination with cisplatin and 5-fluorouracil to treat recurrent or metastatic nasopharyngeal carcinoma. Ann Oncol. 2013;24:1055-61.

8. Cho W, Ziogas DE, Katsios C, Roukos DH. Emerging personalized oncology: sequencing and systems strategies. Future Oncol. 2012;8:637-41.

9. Hu C, Wei W, Chen X, Woodman CB, Yao Y, Nicholls JM, et al. A global view of the oncogenic landscape in nasopharyngeal carcinoma: an integrated analysis at the genetic and expression levels. PLoS One. 2012;7:e41055.

10. Di Fiore F, Blanchard F, Charbonnier F, Le Pessot F, Lamy A, Galais MP, et al. Clinical relevance of KRAS mutation detection in metastatic colorectal cancer treated by Cetuximab plus chemotherapy. Br J Cancer. 2007;96:1166-9.

11. Magnin S, Viel E, Baraquin A, Valmary-Degano S, Kantelip B, Pretet JL, et al. A multiplex SNaPshot assay as a rapid method for detecting KRAS and BRAF mutations in advanced colorectal cancers. J Mol Diagn. 2011;13:485-92.

12. Jiang N, Liu N, Yang F, Zhou Q, Cui R, Jiang W, et al. Hotspot mutations in common oncogenes are infrequent in nasopharyngeal carcinoma. Oncol Rep. 2014;32:1661-9.

13. Izquierdo MA, Van der Valk P, Van Ark-Otte J, Rubio G, Germa-Lluch JR, Ueda $\mathrm{R}$, et al. Differential expression of the c-kit proto-oncogene in germ cell tumours. J Pathol. 1995:177:253-8

14. Bar-Sela G, Kuten A, Ben-Eliezer S, Gov-Ari E, Ben-Izhak O. Expression of HER2 and C-KIT in nasopharyngeal carcinoma: implications for a new therapeutic approach. Mod Pathol. 2003;16:1035-40.
15. Sheu LF, Lee WC, Lee HS, Kao WY, Chen A. Co-expression of c-kit and stem cell factor in primary and metastatic nasopharyngeal carcinomas and nasopharyngeal epithelium. J Pathol. 2005;207:216-23.

16. Bar-Sela G, Ben AM, Sabo E, Kuten A, Minkov I, Ben-Izhak O. Pediatric nasopharyngeal carcinoma: better prognosis and increased c-Kit expression as compared to adults. Pediatr Blood Cancer. 2005;45:291-7.

17. Huang PY, Hong MH, Zhang X, Mai HQ, Luo DH, Zhang L. C-KIT overexpression and mutation in nasopharyngeal carcinoma cell lines and reactivity of Imatinib on these cell lines. Chin J Cancer. 2010;29:131-5.

18. Nishida T, Shirao K, Sawaki A, Koseki M, Okamura T, Ohtsu A, et al. Efficacy and safety profile of imatinib mesylate (ST1571) in Japanese patients with advanced gastrointestinal stromal tumors: a phase II study (ST1571B1202). Int J Clin Oncol. 2008;13:244-51.

19. Demetri $G D$, von Mehren $M$, Blanke $C D$, Van den Abbeele $A D$, Eisenberg $B$, Roberts PJ, et al. Efficacy and safety of imatinib mesylate in advanced gastrointestinal stromal tumors. N Engl J Med. 2002;347:472-80.

20. Cancer Genome Atlas Research Network. Kinase mutations and imatinib response in patients with metastatic gastrointestinal stromal tumor. Nature. 2003;21:4342-9.

21. Inokuchi K, Yamaguchi H, Tarusawa M, Futaki M, Hanawa H, Tanosaki S, et al. Abnormality of c-kit oncoprotein in certain patients with chronic myelogenous leukemia-potential clinical significance. Leukemia. 2002;16:170-7.

22. Foster R, Byrnes E, Meldrum C, Griffith R, Ross G, Upjohn E, et al. Association of paediatric mastocytosis with a polymorphism resulting in an amino acid substitution (M541L) in the transmembrane domain of c-KIT. Br J Dermatol. 2008;159:1160-9.

23. Chua DT, Nicholls JM, Sham JS, Au GK. Prognostic value of epidermal growth factor receptor expression in patients with advanced stage nasopharyngeal carcinoma treated with induction chemotherapy and radiotherapy. Int J Radiat Oncol Biol Phys. 2004;59:11-20.

24. Pan J, Kong L, Lin S, Chen G, Chen Q, Lu JJ. The clinical significance of coexpression of cyclooxygenases-2, vascular endothelial growth factors, and epidermal growth factor receptor in nasopharyngeal carcinoma. Laryngoscope. 2008;118:1970-5.

25. Soo R, Putti T, Tao Q, Goh BC, Lee KH, Kwok-Seng L, et al. Overexpression of cyclooxygenase-2 in nasopharyngeal carcinoma and association with epidermal growth factor receptor expression. Arch Otolaryngol Head Neck Surg. 2005;131:147-52.

26. Ma BB, Poon TC, To KF, Zee B, Mo FK, Chan CM, et al. Prognostic significance of tumor angiogenesis, Ki 67, p53 oncoprotein, epidermal growth factor receptor and HER2 receptor protein expression in undifferentiated nasopharyngeal carcinoma-a prospective study. Head Neck. 2003:25:864-72.

27. Pan J, Tang T, Xu L, Lu JJ, Lin S, Qiu S, et al. Prognostic significance of expression of cyclooxygenase-2, vascular endothelial growth factor, and epidermal growth factor receptor in nasopharyngeal carcinoma. Head Neck. 2013;35:238-47.

28. Lee SC, Lim SG, Soo R, Hsieh WS, Guo JY, Putti T, et al. Lack of somatic mutations in EGFR tyrosine kinase domain in hepatocellular and nasopharyngeal carcinoma. Pharmacogenet Genomics. 2006;16:73-4.

29. Zhang ZC, Fu S, Wang F, Wang HY, Zeng YX, Shao JY. Oncogene mutational profile in nasopharyngeal carcinoma. Onco Targets Ther. 2014;7:457-67.

30. Chua DT, Wei WI, Wong MP, Sham JS, Nicholls J, Au GK. Phase II study of gefitinib for the treatment of recurrent and metastatic nasopharyngeal carcinoma. Head Neck. 2008:30:863-7.

31. Lin L, Bivona TG. Mechanisms of resistance to epidermal growth factor receptor inhibitors and novel therapeutic strategies to overcome resistance in NSCLC patients. Chemother Res Pract. 2012;2012:817297.

32. Janne PA, Ramalingam SS, Yang JC-H, Ahn M-J, Kim D-W, Kim S-W, et al. Clinical activity of the mutant-selective EGFR inhibitor AZD9291 in patients (pts) with EGFR inhibitor-resistant non-small cell lung cancer (NSCLC). J Clin Oncol. 2014;32:5s. suppl; abstr 8009^).

33. Sequist LV, Soria J-C, Gadgeel SM, Wakelee HA, Camidge DR, Varga A, et al. First-in-human evaluation of CO-1686, an irreversible, highly selective tyrosine kinase inhibitor of mutations of EGFR (activating and T790M). J Clin Oncol. 2014;32:5s. suppl; abstr 8010^.

34. Mendoza MC, Er EE, Blenis J. The Ras-ERK and PI3K-mTOR pathways: cross-talk and compensation. Trends Biochem Sci. 2011;36:320-8.

35. Fendri A, Khabir A, Mnejja W, Sellami-Boudawara T, Daoud J, Frikha M, et al. PIK3CA amplification is predictive of poor prognosis in Tunisian patients with nasopharyngeal carcinoma. Cancer Sci. 2009;100:2034-9. 
36. Jiang H, Fan D, Zhou G, Li X, Deng H. Phosphatidylinositol 3-kinase inhibitor (LY294002) induces apoptosis of human nasopharyngeal carcinoma in vitro and in vivo. J Exp Clin Cancer Res. 2010;29:34.

37. Ma BB, Lui WW, Hui CW, Lau CP, Wong CH, Hui EP, et al. Preclinical evaluation of the AKT inhibitor MK-2206 in nasopharyngeal carcinoma cell lines. Invest New Drugs. 2013;31:567-75.

38. Kratz CP, Schubbert S, Bollag G, Niemeyer CM, Shannon KM, Zenker M. Germline mutations in components of the Ras signaling pathway in Noonan syndrome and related disorders. Cell Cycle. 2006;5:1607-11.

39. Fernandez-Medarde A, Santos E. Ras in cancer and developmental diseases. Genes Cancer. 2011;2:344-58

40. Anghel I, Anghel AG, Dumitru M, Soreanu CC. Nasopharyngeal carcinoma - analysis of risk factors and immunological markers. Chirurgia (Bucur). 2012;107:640-5.

41. Zuo Q, Shi M, Chen J, Liao W. The Ras signaling pathway mediates cetuximab resistance in nasopharyngeal carcinoma. Biomed Pharmacother. 2011;65:168-74.

\section{Submit your next manuscript to BioMed Central and take full advantage of:}

- Convenient online submission

- Thorough peer review

- No space constraints or color figure charges

- Immediate publication on acceptance

- Inclusion in PubMed, CAS, Scopus and Google Scholar

- Research which is freely available for redistribution 